\title{
Experimental investigation of upstream cube effects on the wake of a wall-mounted cylinder: Wake rising reduction, TKE budget and flow organization
}

\author{
Magnier Maelys ${ }^{1,2}$, Druault Philippe ${ }^{1,{ }^{*}}$, Germain Gregory 2
}

1 Sorbonne Université, CNRS, UMR 7190, Institut Jean Le Rond d'Alembert, F-75005 Paris, France

2 Ifremer, Marine Structure Laboratory, 150 Quai Gambetta 62200 Boulogne sur Mer, France

* Corresponding author : Philippe Druault, email address : philippe.druault@sorbonne-universite.fr

\begin{abstract}
:
The development of Marine Renewable Energy based on tidal currents at a selected area needs to first analyze the flow characteristics and thus the bathymetry induced turbulence in this area. The present study aims to investigate turbulence characteristics around two wall mounted obstacles in tandem, which represent seabed obstacles in a high Reynolds number flow. 2D planar PIV measurements are performed in multiple planes to access the main flow organization. Based on successive analyses of mean flow characteristics as well as of the flow organization, it is demonstrated that the presence of an upstream cube clearly impacts the cylinder wake development by preventing the rising of the large scale flow structures observed for an isolated wall-mounted cylinder in the same flow conditions. The TKE budget analysis shows that the energy is mainly produced in the upstream cube shear layer, and TKE exchanges are very small in the far wake field, in comparison with a single cylinder flow configuration. A Fourier analysis shows that the cube-cylinder interaction generates a low frequency peak in the far wake field which persists for long downstream distances. This particular frequency is the signature of the cylinder wake coherent structures. A statistical analysis based on the two-point correlation velocity tensor confirms that energetic flow structures present in the far wake are mainly of 2D nature and develop horizontally. Elongated structures are identified in the wake and seem to correspond to hairpin-like structures. Instantaneous vortex structures are also characterized with local vortex identification swirling strength criterion, confirming previous FFT and statistical results. The impact of the upstream cube is strongest in the near wake in the symmetrical transverse plane and prevents the large scale flow structures from rising in the water column.
\end{abstract}

\section{Highlights}

- Wall-mounted cube and cylinder in tandem flow configuration. Characterization of bathymetry induced turbulence. 2D Planar PIV measurements of the wake interference. Spectral, statistical and instantaneous characterization of the large scale flow organization Turbulent Kinetic Energy budget.

Keywords : PIV measurements, Wake interference, Flow structure organization, Vortex Shedding 


\section{Introduction}

Nowadays, the increased energy requirement as well as the problems of climate change leads to develop some new renewable energies. Among them, the Marine Renewable Energy (MRE) based on tidal currents is a green energy source, emitting no greenhouse gases. However, the development of such a MRE needs the inflow variation effects among bathymetry induced turbulence to be investigated at first. Each potential tidal area being unique, a detailed analysis is necessary to study the tidal energy device life-time. A lot of work has been done in this way for specific turbulent inflow characteristics $[1,2,3]$. One of the worst effects on tidal turbine is due to the presence of large scale energetic flow structures induced by bathymetry variations.

The interaction of high-speed flow with irregular roughness bathymetry or isolated roughness elements located at the seabed creates and develops flow structures (potentially periodic or the rare burst one) which could notably impact the performance of the turbine (structural load, blade fatigue, ....).

The turbulent flow over rough surfaces has also been extensively studied with various different roughness elements as a function of industrial and environmental applications. The first simplest geometry is the single wall-mounted cube which has been extensively studied experimentally and numerically. This benchmark test case was often considered due to the underlying complex flow dynamics including three-dimensional separation, recirculation area, horseshoe vortex formation, vortex shedding in the shear layer $[4,5,6,7]$. Indeed, it was demonstrated 
that unsteady flow separation generates organized structures downstream of the cube (hairpin and vortex shedding structures), which are generally regularly dissipated in the far wake [6]. Note that when the cube is immersed in a turbulent boundary layer flow, it was shown that low frequency structures can persist far downstream from of the cube [8], but these structures do not rise in the water column, even in presence of an inclined floor behind the cube [7]. Consequently, the cube wake flow is only developed over a small vertical extent, and large scale flow structures dissipate in the far streamwise direction.

On the other hand, surface-mounted 2D elements (like rib, horizontal cylinder or dune) were also often used to represent simplified roughness elements $[9,10,4,11,12]$. The wake results of a wall-mounted cylinder highlighted that Kelvin-Helmholtz vortices are generated periodically from the upstream edge of the obstacle and they fully interact or merge to create downstream hairpin structures $[10,13,14]$. These large scale periodic structures (which are sometimes called kolk-vortices) then rise in the water column in the far wake $[15,12]$. Contrary to cube wake results, this highlights that bathymetry in presence of 2D obstacles (dune or cylinder-like element) generates large scale structures which potentially have negative effects on the tidal turbine positioned in the wake [16].

The studies of isolated canonical configurations gave a lot of interesting results but they need to be extended to more realistic configurations. Indeed, turbulent flow over two (or more) roughness elements in tandem or in close proximity (more representative to seabed variations) have been less studied than those related to one isolated roughness element and have to be considered. In the presence of at least two obstacles, the mutual interaction of the flow wakes leads to quite complex flow dynamics but the understanding of such interactions is essential to numerous environmental and industrial applications. The resulting experimental database is also of great importance to validate numerical models like the one developed by Mercier et al. [14].

Note that flow around two slender structures in tandem has been the subject of extensive research due to its practical significance in many engineering applications. A lot of previous studies have then mainly focused on the wake dynamics of two tandem circular slender cylinders emphasizing the complex flow physics: flow separation, quasi-periodic vortices, shear layer development, vortex shedding interaction, ... [17, 18]. It was demonstrated that flow dynamics strongly depend on multiple parameters such as the nature of the flow (Reynolds number, inflow turbulent boundary layer), the cylinder spacing and height ratio [19, 20, 18], the body diameter 
[21]. But the underlying flow dynamics differ from the one arising from specific bathymetry roughness such as wall mounted horizontal obstacles.

Flow over wall mounted cubes in tandem, immersed in a laminar boundary layer, has been investigated by Martinuzzi and Havel [22]. They showed distinct behaviors depending on the cube spacing due to the modification of the shear layer curvature developing over the first cube. For intermediate spacing $(1.5<S / H<2.5$, where $S$ and $H$ are the cube spacing and height, respectively), the shedding frequency of the flow structures developed in the wake scales inversely with the cube spacing [23]. Very few studies have dealt with 2D wall-mounted elements in tandem, numerical simulations only have been performed recently but in a 2D context [8, 24].

In the present work, a new combination of a wall-mounted element in tandem is considered: a cube followed by a cylinder of aspect ratio of 6 , both with the same height. The main focus is then to quantify the effect of the cube presence on the wake development of the wall-mounted cylinder and on the energy exchange between the two wakes via the kinetic energy budget. The wake characterization obtained on an isolated cylinder [12] as well as on an isolated cube [7] will serve as a baseline of comparison for the present investigation.

After a short description of the experimental set-up, the mean flow characteristics and the turbulence statistics will be described from 2D PIV measurement results. Then, the analysis of the wake organization is conducted by extracting three complementary quantities : i) a Fourierbased frequency analysis, ii) a statistical analysis by considering a two-point correlation tensor of the velocity field and iii) an instantaneous analysis based on a vortex identification swirling strength criterion. Finally, the spatial distribution of the turbulent energy budget will be studied in order to show how the presence of the cube modifies the wake energy exchanges of the present tandem configuration.

\section{Experimental facilities presentation}

Tests are carried out in the wave and current circulating flume tank of IFREMER located in Boulogne-sur-Mer (France) [25]. The test section is $18 \mathrm{~m}$ long $\times 4 \mathrm{~m}$ wide $\times 2 \mathrm{~m}$ deep. The incoming flow $\left(U_{\infty}, V_{\infty}, W_{\infty}\right)$ is assumed to be steady and constant, with $U_{\infty}=1 \mathrm{~m} \cdot \mathrm{s}^{-1}$ and $V_{\infty}=W_{\infty}=0$. The three instantaneous velocity components are denoted $(U, V, W)$ along the $(x, y, z)$ directions respectively. According to the Reynolds decomposition, each instantaneous 
velocity component is separated into a mean value and a fluctuating part: $U=\bar{U}+u^{\prime}$ where the overbar indicates the time average and $\overline{u^{\prime}}=0$. By the use of a grid combined with a honeycomb placed at the inlet of the working section, the upstream flow turbulence intensity is: $I_{\infty}=1.5 \%$.

For the present investigation, the incoming flow Reynolds number is $R e=\frac{H U_{\infty}}{v}=2.5 \times 10^{5}$ with $H$ the roughness height and $v$ the water kinematic viscosity. The Froude number is $F r=$ $\frac{U_{\infty}}{\sqrt{g D_{e}}}=0.23$ with $D_{e}$ the flume tank depth and $g$ the gravity. So the scaled experiment has non-dimensioned parameters similar to those of real sea conditions [12], which correspond to Alderney Race conditions, a potential future tidal site in French water.

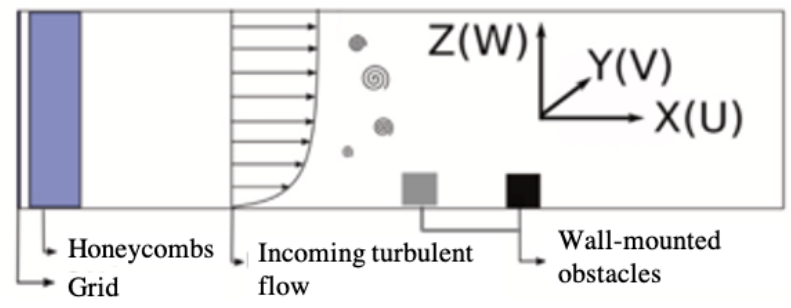

Figure 1: Schematic view of the experimental set-up.

To characterize the flow, planar Particle Image Velocimetry (PIV) is used. Beforehand, the tank is seeded with $10 \mu \mathrm{m}$ diameter silver coated glass micro-particles. A particle is detected on 3 to 5 pixels. Cross-correlation is used to calculate particle displacement between two images and outliers are replaced with the Universal Outlier Detection method [26]. PIV acquisitions are made during $150 \mathrm{~s}$, hence 2250 double images are taken with a $15 \mathrm{~Hz}$ acquisition frequency. Plane dimensions are $1153 \times 430 \mathrm{~mm}^{2}$ with a spatial resolution of $d x=d z=1.6 \mathrm{~mm}$. Depending on the plane, from $1 \%$ to $13 \%$ of the total vectors number are substituted. Various sources of experimental errors can be identified. Positions fixed using the human eye are considered to have a maximum error of $0.5 \mathrm{~cm}$ and $0.5^{\circ}$; it is the case for the obstacle and the lasers positioning. PIV calibration is precise at $1 \mathrm{~mm}$. The pixel size is added to the errors for PIV measurements. Hence, the errors on the results are of $2.6 \%$ [12].

The wall mounted obstacles studied are presented in figure 2. Cube side measures $H=0.25 \mathrm{~m}$ and $H$ is chosen as reference length for the normalization. The following notations will be used: $x^{*}=x / H, y^{*}=y / H$ and $z^{*}=z / H$. The cylinder section is a square of side $H$. It is $6 H$ long, corresponding to an aspect ratio of 6 . The element spacing is fixed to $2 H$ and the cube is located upstream of the cylinder. Such a spacing allows the shear layer separated from 
the cube to reattach on the downstream cylinder [23, 27]. The $x$-origin is set at the center of the cylinder (figure 2). $z^{*}=0$ corresponds to the wall. Obstacles are disposed symmetrically around spanwise origin $y^{*}=0$. PIV measurements are carried out at various transverse positions: $y^{*}=0, y^{*}=1$ and $y^{*}=2$ (denoted $y 0, y 1$ and $y 2$ respectively) represented in figure 2-right. In each spanwise plane, PIV measurements are performed on 14 PIV planes. Figure 2 presents their spatial organization in the symmetrical median plane $y 0$ which is quite similar to the other two spanwise planes. At the cube position, the boundary layer height is calculated as follows $\delta=\delta_{95}=z\left(\bar{U}=0.95 \times U_{\infty}\right)$, is equal to $\delta=1.3 H$.

\section{Mean flow statistics and Turbulent Kinetic Energy balance}

Side view (y0-plane)

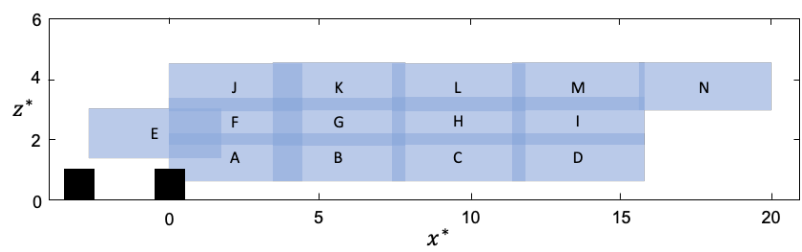

Top view

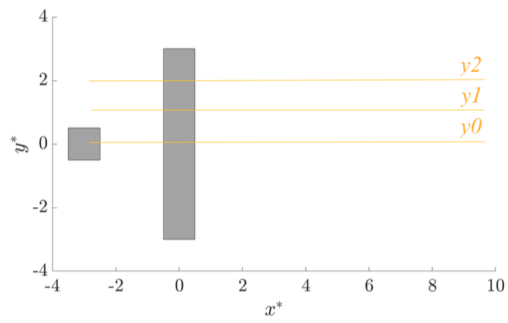

Figure 2: PIV measurement planes locations in the $y 0$-plane (on the left) and schematic representation of the tandem flow configuration with the positions of the spanwise planes, $y 0, y 1$ and $y 2$ (on the right).

To characterize the flow around the obstacles, mean flow properties will be studied (streamwise and vertical velocity fields, Turbulent Kinetic Energy - TKE defined as $k=\frac{1}{2}\left(\overline{u^{\prime 2}}+\overline{w^{\prime 2}}\right)$. First, in order to emphasize the modifications of the cylinder wake recirculation area in presence of an upstream cube, a method based on Proper Orthogonal Decomposition for reconstructing the mean flow field in the 3D space is implemented and presented in the following. Second, an analysis of the TKE budget is done. TKE energy budget equations are then recalled and the dissipation rate estimation is described. After the description of the associated post-processing tool implementation, mean flow results as well as TKE energy budget are provided. 


\subsection{Post-processing tools implementation and TKE budget equations}

POD method implementation for the $3 D$ mean velocity reconstruction

The method applied to plane $A$ measurement is briefly presented. Details about the application of the method are given in [28, 29].

Instantaneous velocity fields extracted in plane $A$ for each transverse plane are put in a single matrix $U=\left[u^{A}(x, z, y 0, t), u^{A}(x, z, y 1, t), u^{A}(x, z, y 2, t)\right]$ of size $\left[N_{x} \times N_{z}, N_{y} \times N_{t}\right]$. A POD is then applied to this matrix provided $N_{\text {mod }}=N_{x} \times N_{z}$ POD spatial eigenfunctions $\Phi^{(n)}(x, z)$ of size $\left(N_{x}, N_{z}\right)$ and $N_{\text {mod }}$ POD temporal coefficients $a^{(n)}(t)$ of size $\left(N_{t} \times N_{y}\right)$ deduced from the projection of $U$ onto $\Phi^{(n)}(x, z)$. These temporal coefficients are then reorganized as follows: $\left[a_{0}\left(y 0,1: N_{t}\right) a_{1}\left(y 1,1: N_{t}\right) a_{2}\left(y 2,1: N_{t}\right)\right]$ where each vector $a_{i}$ is of size $N_{t}$. POD leads to the following exact reconstruction:

$$
u^{A}\left(x, z, y_{i}, t\right)=\sum_{n=1}^{N_{\text {mod }}} a_{i}^{(n)}(t) \Phi^{(n)}(x, z)
$$

with $i=0,1,2$. Then a reduced number of POD modes called $M$ is chosen allowing to best fit the Reynolds average mean velocity field: $\overline{U^{A}\left(x, z, y_{i}\right)}=\sum_{n=1}^{M} \overline{a_{i}^{(n)}} \Phi^{(n)}(x, z)$ in each yi-plane. In the present work like in [29], $M=5$ is retained so as to best approach the mean flow field in each of the three $y \mathrm{i}$-planes because in all these planes, the average value of the $5^{t} h$ POD coefficient is always close to zero (lower than $10^{-3}$ ). Then, a yi-spatial cubic spline interpolation (between the three PIV measurement planes) of each temporal mean POD temporal coefficient is performed. The temporal coefficients are interpolated at any $y$ location between $y 0$ and $y 2\left(y^{*} \in[0: 2]\right)$ They are denoted $\overline{a^{(n) r e c .}(y)}$ with $n \in[1: M]$. Finally, the reconstruction of the mean flow in the 3D space can be carried out as follows:

$$
\overline{U^{A}(x, y, z)}=\sum_{n=1}^{M} \overline{a^{(n) r e c} \cdot(y)} \Phi^{(n)}(x, z) .
$$

\section{TKE energy budget equations}

For an incompressible Newtonian fluid, the governing equations for the fluid movements are the equations of continuity and the Navier-Stokes equations. With $k=\frac{1}{2} \overline{u_{i}^{\prime} u_{i}^{\prime}}$ the turbulent kinetic energy, [30] described the turbulent kinetic energy budget as follows:

$$
\frac{\partial k}{\partial t}+\underbrace{\overline{U_{i} \frac{\partial k}{\partial x_{i}}}}_{C}=-\underbrace{\frac{1}{\rho} \frac{\partial}{\partial x_{i}} \overline{u_{i}^{\prime} p^{\prime}}}_{\Phi}-\underbrace{\frac{1}{2} \frac{\partial}{\partial x_{j}} \overline{u_{i}^{\prime} u_{i}^{\prime} u_{j}^{\prime}}}_{T}-\underbrace{\overline{u_{i}^{\prime} u_{j}^{\prime} \frac{\partial \overline{U_{i}}}{\partial x_{j}}}}_{P}+\underbrace{v \frac{\partial \overline{\left(\frac{\partial u_{i}^{\prime} \partial u_{j}^{\prime}}{\partial x_{i}}\right)}}{\left.\partial x_{j}\right)}}_{D_{\mu}}-\underbrace{v \overline{\left(\frac{\partial u_{i}^{\prime}}{\partial x_{j}}+\frac{\partial u_{j}^{\prime}}{\partial x_{i}}\right) \frac{\partial u_{j}^{\prime}}{\partial x_{i}}}}_{\varepsilon}
$$


Equation (3) means that temporal variations of kinetic energy $k$ are linked to:

- $C$, the energy convection due to movement of molecules in fluids,

- $\Phi$, the energy transport due to pressure effect,

- $T$, the energy transport due to the vortices movement,

- $P$, the transfer of energy from the average movement to the turbulent movement (production),

- $D_{\mu}$, the viscous diffusion,

- and $\varepsilon$, the viscous dissipation.

This equation is of great help to examine the spatial distribution of turbulent energy. It helps also to characterize the different mechanisms involved and whether the turbulent energy feeds large or small scales. In our case, planar PIV measurements give only vertical and streamwise velocity components. Thus, one restricts the TKE budget analysis to the symmetrical $y 0$-plane where the flow is $2 \mathrm{D}$, that means $\bar{V}=0$ and $\frac{\partial}{\partial y} \overline{(.)}=0$. In this case, the kinetic energy $k$ is expressed as follows [31]: $k=\frac{3}{4}\left(\overline{u^{\prime 2}}+\overline{w^{\prime 2}}\right)$. Moreover, for high Reynolds number, $D_{\mu}$ is negligible and $\overline{v^{\prime 2} w^{\prime}}=\overline{w^{\prime 3}}, \overline{v^{\prime 2} u^{\prime}}=\overline{u^{\prime 3}}$ [32]. Following [33] method, equation (3) becomes:

$$
\begin{aligned}
\frac{\partial k}{\partial t}+\underbrace{\bar{U} \frac{\partial k}{\partial x}+\bar{W} \frac{\partial k}{\partial z}}_{C}=-\Phi- & (\underbrace{(\frac{1}{2} \frac{\left.\partial \overline{u^{\prime 2} w^{\prime}}+\frac{\partial}{\partial z} \overline{w^{\prime 3}}+\frac{1}{2} \frac{\partial}{\partial x} \overline{u^{\prime 3}}+\frac{\partial}{\partial x} \overline{u^{\prime} w^{\prime 2}}\right)}{\left(\overline{u^{\prime} u^{\prime}} \frac{\partial \bar{U}}{\partial x}+\overline{w^{\prime} w^{\prime}} \frac{\partial \bar{W}}{\partial z}+\overline{u^{\prime} w^{\prime}} \frac{\partial \bar{U}}{\partial z}+\overline{u^{\prime} w^{\prime}} \frac{\partial \bar{W}}{\partial z}\right)}-\underbrace{15 v\left(\frac{\partial u^{\prime}}{\partial x}\right)^{2}}_{P}}_{T}
\end{aligned}
$$

As $D_{\mu}$ is neglected, the rest of this equation is considered to be equal to the pressure gradient term $\Phi$. Partial derivatives are calculated using a second order centered finite difference method. Dissipation occurs from the Kolmogorov length scale $\eta$ [34], and due to the PIV mesh resolution (equal to $11.6 \mathrm{~mm}$ ), dissipation cannot be calculated directly. Following previous recommendations [32], the dissipation rate is estimated using the Large Eddy-PIV (LE-PIV) method [35]. Even if this LE-PIV method based on a spatial scale filtering separation within the inertial range tends to slightly under-estimate the dissipation rate [32], it provides a good approximation of this rate. 


\subsection{Results}

\section{Mean flow characterization}

Figure 3 presents the mean streamwise and vertical velocity components in the median $y 0$ plane and in $y 2$-plane. In the $y 0$-plane (figure 3 (top)), the flow separates at the leading edge of the cube. Compared to an isolated cube wake [7], the associated shear layer is forced to accelerate over the cylinder. Behind the cylinder, in the centerline plane, the recirculation area (calculated by extrapolating the zero mean streamwise velocity contour that meets the wall) is greatly reduced owing to the presence of the upstream cube, by comparison with the recirculation area obtained from an isolated cylinder in similar flow conditions [12]. Such a result is similar to those obtained in the wake of two wall-mounted small vertical cylindrical structures in tandem for similar cylinder spacing [27, 20], where a more compact recirculation area is observed in the centerline plane behind the second obstacle. Based on the mean flow representation (figure 3), the recirculation length is $l_{y 0}^{*}=2.3$, calculated from the center of the bottom face of the cylinder. This value is two times smaller than the one obtained by [4] for a cylinder alone with similar Reynolds number flow and cylinder aspect ratio. The cube breaks the mean wake development of the cylinder.

Looking at figure 3 (bottom), in the $y 2$-plane, the flow has the classical behavior of a wake flow behind a square cylinder as presented in [12]. It separates at the leading edges of the cylinder in an outer steady region and a recirculation area downstream of the cylinder. A shear layer develops in-between. The flow reattaches after the recirculation region. In this $y 2$-plane, the cylinder edge located at $y^{*}= \pm 3 H$ ) effects dominate the cube influence, which seems to have no impact on the mean cylinder wake. In this plane, the recirculation length measures $l_{y 2}^{*}=4.1$, while Ikhennicheu et al. [12] obtained $l_{y 2}^{*}=4.5$ for an isolated structure. Both values are similar, confirming that the cube has little influence on the wake dynamics in this transverse plane.

Above the black line (corresponding to $\bar{U}=0.90 * U_{\infty}$ ), for $-0.5<x^{*}<6$, the mean flow is accelerated. It reaches $\bar{U}=1.19 \mathrm{~m} . \mathrm{s}^{-1}$ in the $y 2$-plane. This overspeed still exists on top of the wake of a single wall-mounted square cylinder [12] but here it persists in the flow. Whatever the spanwise position $y$, the flow has a strong vertical motion on top of the cylinder. Then in the far field, the mean vertical velocity component exhibits negative values meaning that the mean flow field tends to move towards to the wall.

As the mean flow field is symmetrical with respect to the symmetrical $y 0$-plane, the final 


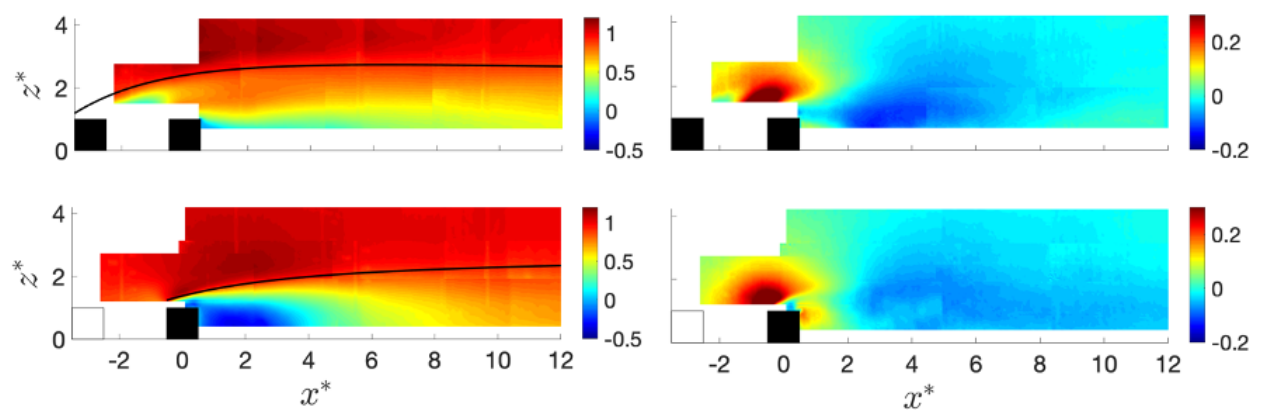

Figure 3: Mean streamwise velocity $\bar{U}$ (left). Mean vertical velocity $\bar{W}$ (right). Top: in $y 0$-plane. Bottom: in $y 2$-plane Continuous black lines correspond to lines where $\bar{U}=0.90 * U_{\infty}$.

mean flow is reconstructed within the interval $y^{*} \in[-2: 2]$. An illustration of the mean 3D flow reconstruction is displayed in figure 4 from measurements in areas $A$ and $F$. Three iso-surface values $\left(1 \mathrm{~m} \cdot \mathrm{s}^{-1}, 0.1 \mathrm{~m} \cdot \mathrm{s}^{-1}\right.$ and $\left.-0.1 \mathrm{~m} \cdot \mathrm{s}^{-1}\right)$ of the mean velocity field are represented in order to confirm the impact of the upstream cube on the cylinder wake recirculation area which is greatly reduced in the centerline plane $\left(y^{*}=0\right)$. Moreover, the flow acceleration due to the cube shear layer development is also clearly indicated along the $x$ direction for $y^{*} \in[-1: 1]$.
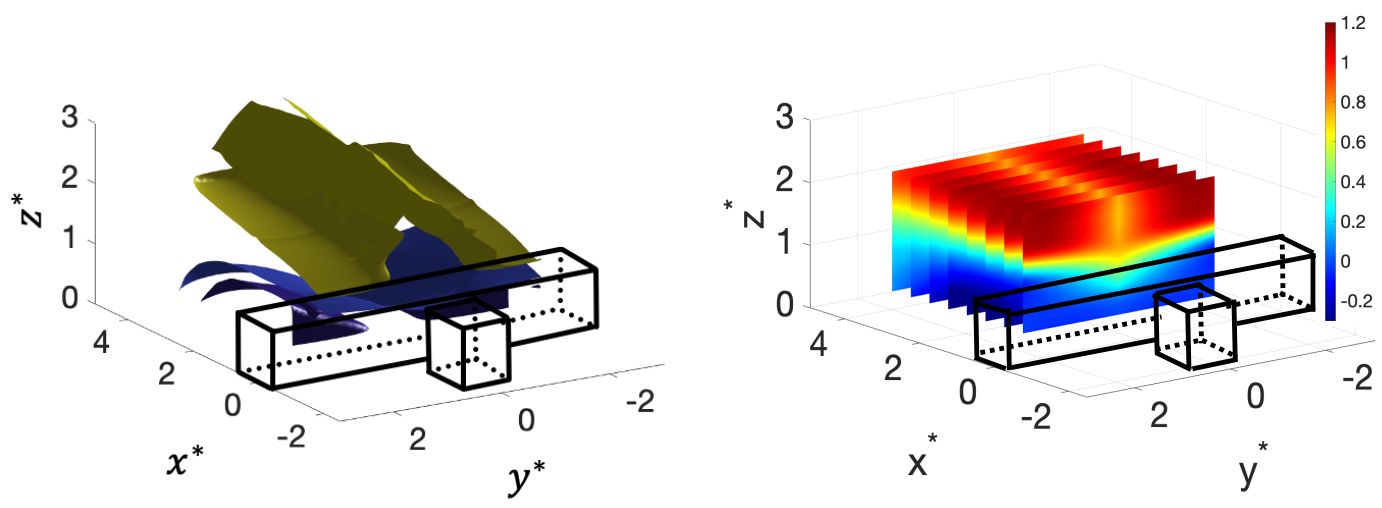

Figure 4: Reconstructed mean streamwise velocity component in 3D space.

Left: Isosurfaces values: $1 \mathrm{~m} \cdot \mathrm{s}^{-1}$ (yellow), $0.1 \mathrm{~m} \cdot \mathrm{s}^{-1}$ (blue), $-0.1 \mathrm{~m} \cdot \mathrm{s}^{-1}$ (purple) from measurements in areas A and $\mathrm{F}$. Right: 8 slices from $x^{*}=0.5$ to $x^{*}=4$ showing the $x$ variation of the mean flow magnitude, from measurement in area A. 


\section{Turbulent kinetic energy budget}

2D Turbulent Kinetic Energy is a marker of the extension of turbulent perturbation in the wake and gives an evaluation of the turbulent content produced in the flow, especially from the turbulent energy budget.

In each measurement plane, one extracts $k$ along selected vertical lines for which $x$ corresponds to the center of each measurement plane. So, at a fixed streamwise position, the $z$ profile of the TKE is obtained from TKE calculated in 3 measurement planes (figure 5). For instance, at $x^{*}=2.2$, the $z$-profile of TKE comes from PIV measurement planes $A, F$ and $J$. In the near wake $\left(x^{*}<3\right)$, in the $y 0$-plane, the vertical profiles contain two peaks whose amplitudes decay in the far wake. The first one comes from the shear layer detached from the cube wake $\left(z^{*} \sim 2.5\right)$ and the other one from the cylinder wake $z^{*}<1$. In this $y 0$-plane, the high level TKE area is due to the cube separated shear layer interacting with the cylinder. Further downstream, both shear layers are convecting and quickly merging leading to a peak plateau around $z^{*} \in[1.5: 2.5]$ in the far field. From $x^{*}>10$, this TKE plateau does not rise and remains at similar $z$ positions, $z^{*} \approx 2$. This differs from previous investigations regarding the wake of an isolated cylinder [36] where the TKE maximum rises along the $x$ direction.

In the $y 1$-plane, at $\left(x^{*}, z^{*}\right)=(2.2,0.9)$ and in the $y 2$-plane, at $\left(x^{*}, z^{*}\right)=(2.2,1.25)$, only one dominant TKE peak is observed. These peaks correspond to the cylinder shear layer development. Further downstream, TKE profiles of both $y$-planes exhibit similar behavior where the TKE peaks slightly move vertically and resemble those of the isolated cylinder [36]. In the far field, from $x^{*}>10$, TKE profiles tend to become more homogeneous along the spanwise direction, with a small similar level of TKE observed over a plateau located in the same vertical area.

Globally, the presence of the upstream cube significantly modifies the second order turbulence statistics in the wake of the cylinder, in the symmetrical y0-plane. In this plane, in the near wake, the TKE levels are reduced in comparison with a single cylinder wake flow. This is directly linked to the reduction of the cylinder shear layer development, especially along the vertical direction. These TKE modifications are quite similar to those observed in the near wake of two wall-mounted small vertical cylinders in tandem [20]. Further downstream, the TKE levels seem to relax toward their original unperturbed state, without the upstream cube.

Figure 6 displays the TKE budget in some selected streamwise locations in order to study 

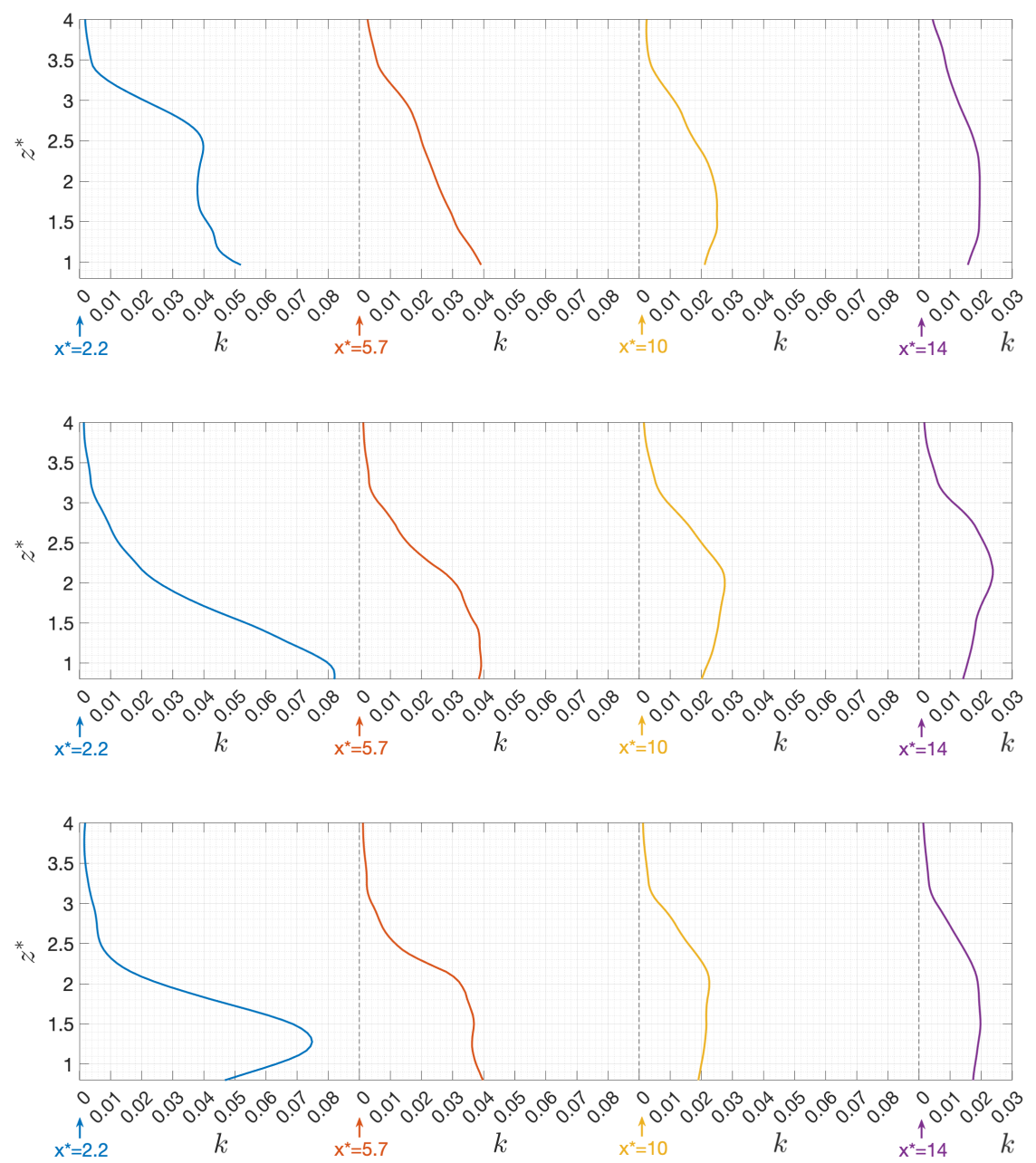

Figure 5: $T K E$ profiles as a function of $z^{*}$ in four different streamwise positions: $x^{*}=2.2, x^{*}=5.7, x^{*}=10, x^{*}=14$. Top: $y 0$-plane. Center: $y 1$-plane. Bottom: $y 2$-plane.

the energy flux exchange between the different flow scale structures of the flow. Globally, the magnitude of all quantities is relatively high in the near wake, compared to the far wake. TKE exchanges are mainly done before $x^{*}=2$. TKE profiles also illustrate the presence of both separated shear layers coming from the cube and the cylinder wake $\left(x^{*}=1\right)$. Then, these shear layers merge, one rising slowly in the water column until $x^{*}=3$, with a decreasing intensity when moving further from the obstacle. Dissipation rate is low and almost constant all over the wake. Convection and pressure effects are evolving the opposite way around. The rising movement 
of their crossing points corresponds to the elevation of the shear layer. TKE is transported by pressure effects under the shear layer and by turbulent motion above.

Finally, TKE is mainly produced in the upstream cube shear layer, from $x^{*}=1$ and TKE exchanges are very small in the far wake field, in comparison with a single cylinder flow configuration [32]. Understanding these energy fluxes is of importance for the future implementation of a tidal energy device by potentially improving the energy exchanges between the flow and the energy converters.
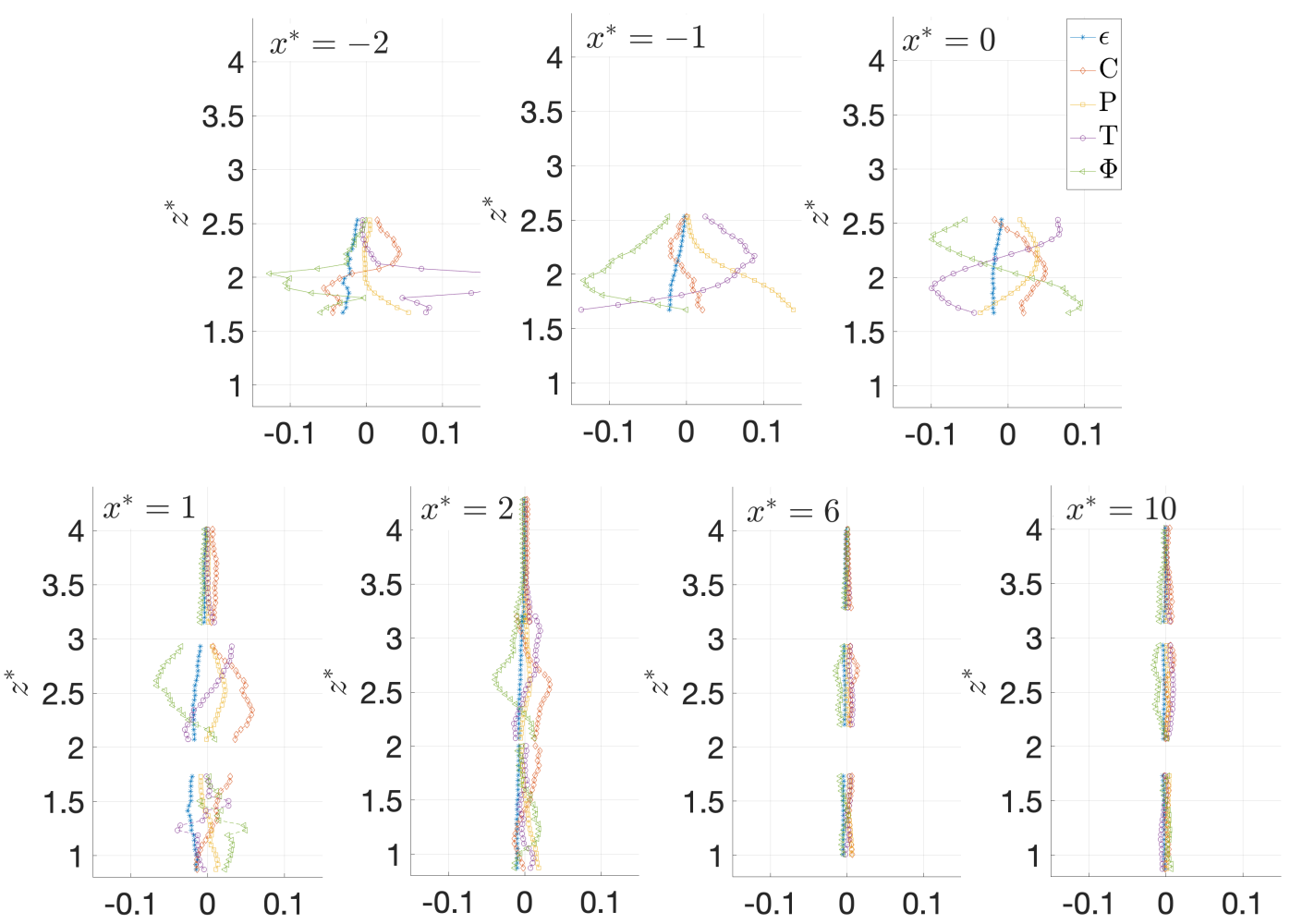

Figure 6: Turbulent kinetic energy budget $z$-profiles at seven $x^{*}$ locations (y0-plane), in near and far wake field. See equation 3 for the definition of the terms: $\epsilon, C, P, T$ and $\Phi$.

\section{Characterization of the wake flow organization}

The wake flow organization is successively analyzed from three viewpoints. First a Fourier analysis is conducted to extract the periodic large scale flow structures. Secondly, a statistical 
analysis is performed from the two-point spatial correlation tensor of the velocity field allowing an estimation of the size and strength of the vortices. Thirdly, instantaneous vortex identification methods are implemented by analyzing the swirling strength criterion.

\subsection{Spectral analysis}

A Fourier analysis is done by watching the Power Spectral Density (PSD) of the fluctuating streamwise velocity component. For all computations presented in this article, each velocity signal is split over window intervals of 1024 points (using a Hanning window) and with 50\% overlap. PSD normalization is the same for all figures presented. Note that when plotting at one point the PSD as function of the Strouhal, the $-5 / 3$ slope characteristic of the inertial range of the energetic cascade of turbulence is observed, confirming thus the dissipation process. To appreciate the streamwise and vertical development of periodic large scale flow structure, PSDs can be presented at a fixed $x^{*}$ position, for all available $z^{*}$. Figure 7 presents, at selected $x^{*}$ located at the middle of each measurement plane (as we did for figure 5), the resulted PSDs expressed as a function of the Strouhal number, $S t=\frac{f H}{U_{\infty}}$ in the $y 0$ and $y 2$-planes. For comparison, a similar analysis is also carried out from a previous study [12] corresponding to the wall-mounted isolated cylinder wake flow in the symmetrical $y 0$-plane (top planes).

First, results deduced from an isolated cylinder wake flow confirm that large scale periodic structures are developed in the wake with a characteristic Strouhal number of 0.07 [12]. The rising of these flow structures in the far wake is clearly indicated as their footprint is present in the upper measurement planes $\left(z^{*}>3.5\right)$. This Strouhal value is similar to previous cylinder wake investigations $[37,15]$. The vortices reach the altitudes $z^{*}>4$ for $x^{*}>19$, while they reach $z^{*} \approx 3.5$ for $8<x^{*}<16$ and stay lower than $z^{*}=3$ for $x^{*}<8$.

Secondly, for the two obstacles present in tandem flow configuration, in the $y 0$ transverse plane (figure 7 (center)), the near cube wake is dominated by a vortex shedding frequency corresponding to a Strouhal number around 0.1. This frequency peak value is similar to previous works dealing with a single cube wake vortex-shedding frequency $[5,6]$. Then, when the cube wake flow interacts with the downstream cylinder, some low frequency peaks are observed. This can be related to two cubes in a tandem flow configuration where similar observations were made [22].

Downstream from the obstacles, even if various frequency peaks are distinguishable due to the 3D flow interactions, $S t \approx 0.07$ peak remains, that is not the case for $S t \approx 0.1$. The cube 


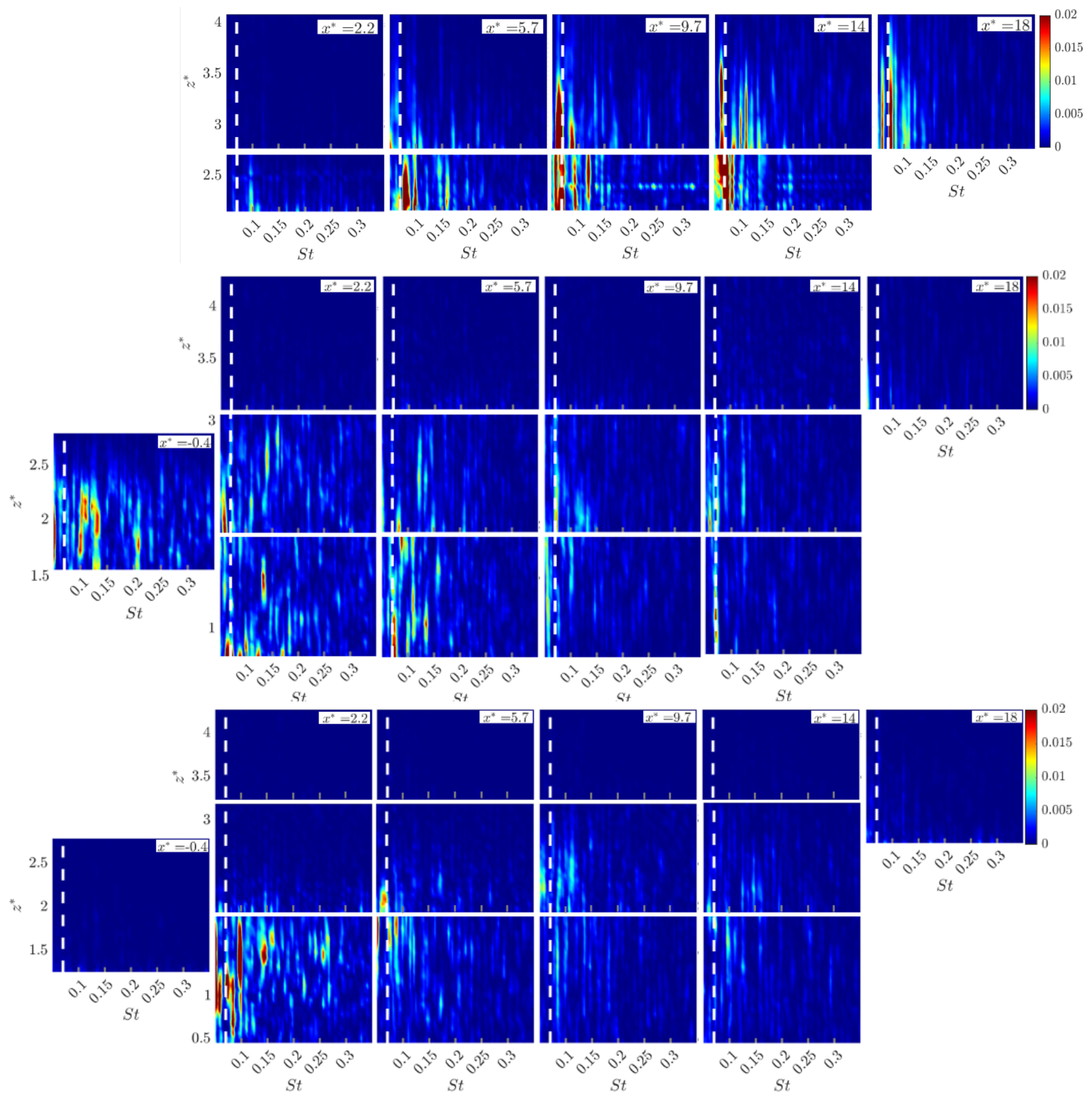

Figure 7: PSD maps of the fluctuating streamwise velocity component, at selected $x^{*}$ located at the middle of each measurement plane. Top: PSD map obtained from previous available database [12] (in the symmetrical $y$-plane of an isolated wall-mounted cylinder). PSD maps related to tandem flow configuration obtained in the $y 0$-plane (center) and in $y 2$-plane (bottom). The white vertical dotted lines indicate $S t=0.07$. 
periodic vortex shedding is annihilated. In the far wake field (in the transverse symmetrical $y 0$ plane), some periodic flow structures characterized by $S t \approx 0.07$ are present and they are related to the same flow structure frequency passage behind an isolated cylinder [12]. However, this frequency peak is only obtained for $z^{*}<3.5$. No periodic flow structures are observed in the upper measurement planes $\left(z^{*}>3.5\right)$. In comparison with the isolated cylinder, PSD results emphasize that similar larger scale structures are developed in the far field, but they do not rise in the water column. They only move horizontally in the far wake.

In the other transverse planes ( $y 1$ (not shown here) and $y 2$ ), the effect of the cube wake is not easily visible and low frequency peaks coming from the cylinder wake vortex shedding are still dominated.

Globally, the interaction between a wall-attached cube followed by a wall mounted cylinder generates a low frequency peak $(S t=0.07)$ in the far wake field which persists for long downstream distances. This corresponds to the signature of the cylinder wake coherent structures. Even if in the symmetrical $y 0$-plane, the near wake is of 3D nature, the cylinder wake seems to re-organize in the far field leading to an apparent $2 \mathrm{D}$ field. The presence of the cube only alters the near wake dynamics in the symmetrical $y 0$-plane. However this interaction leads to the suppression of the vertical motion of the flow structures in the far field.

\subsection{Spatial correlation analysis and flow scales}

The statistical properties of the vortices are now examined from the two-point spatial correlation tensor. The computation of the correlation coefficient of both the streamwise and the vertical velocity components is made. For example, the $R_{u u}$ coefficient is defined as follows:

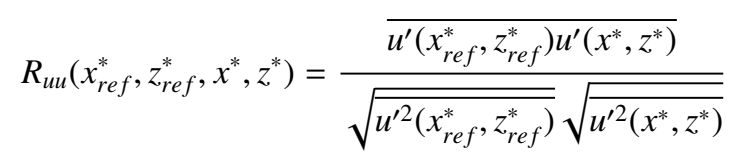

where $\left(x_{r e f}^{*}, z_{r e f}^{*}\right)$ are the coordinates of a fixed reference point. As an illustration, figure 8 presents $R_{i i}(i=u, w)$ map for $\left(x_{r e f}^{*}, z_{r e f}^{*}\right)=(2.6,2.6)$ in the symmetrical $y 0$-plane. $R_{u u}$ is mainly positive but exhibits also negative levels at the bottom of the plane. $R_{w w}$ exhibits a smaller positive area. Thus, vertical motions are statistically less correlated than horizontal ones. Black ellipses materialize the 0.6-contour level. This rather high contour value has been chosen for having statistically good accuracy while focusing on large-scale events [38]. Then to access the spatial development of the coherent structures in each $y$-plane, the isopleth equal to 0.6 is 
deduced from the correlation coefficient maps of two chosen points in each measurement plane and plotted.

Figure 9 presents the resulted 0.6-contour level (corresponding to black isopleth in figure 8) in two points per PIV planes, in $y 0, y 1$ and $y 2 . R_{u u}$ ellipses are elongated in the streamwise direction. 0.6-isopleths of the vertical velocity component are rather round. However, no preferential inclination appears either for $R_{u u}$ or for $R_{w w}$. Isopleths of $R_{u u}$ and $R_{w w}$ exhibit patterns similar to those observed in a turbulent boundary layer. These characteristics are commonly attributed to hairpin structures $[39,40]$ which have also been identified in wall-mounted 2D obstacle wakes $[10,13,14]$. In $y 2$-plane (ellipses in red in the figure), no significative correlation exists in median PIV planes $\left(z^{*} \in[2: 4], x^{*}<8\right)$. Conversely, in the symmetrical y0-plane (black ellipses in the figure), due to the the presence of the cylinder the wake of the cube is shifted above $z^{*}>2$. In $y 1$-plane (blue ellipses in the figure), results in measurement plane $E\left(x^{*} \approx 0\right)$ exhibit some statistical coherent structures whereas there is no correlation stain in PIV plane $F$, just downstream from it. This brings to light side vortices of the cube that are lifted up by the cylinder even if no frequency signature was revealed from Fourier analysis. Similarly, in PIV plane $N\left(x^{*}>16\right)$ non-periodic flow structures are present in the flow at $z^{*}>3$ while no structures are detected at this level for $x^{*}<16$ (see figure 7 (center)). It is also interesting to observe that whatever the $y$-plane, from $x^{*}=9.7$, isopleths become similar. Some large scale organized coherent structures are present. They correspond to those detected from the Fourier analysis at $S t=0.07$ (figure 7).
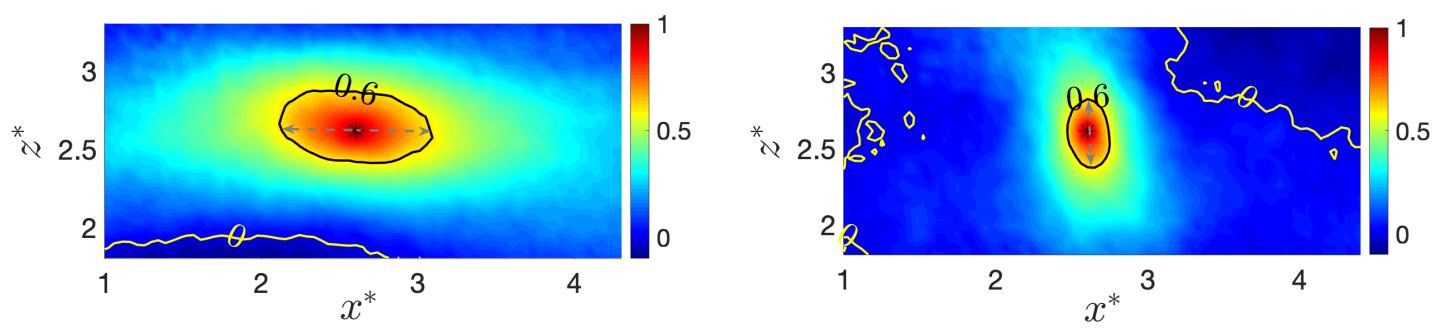

Figure 8: Two-point correlation maps $R_{i i}(i=u$, left $)$ and $(i=w$, right $)$ obtained at a reference point $x_{\text {ref }}^{*}=2.6, z_{\text {ref }}^{*}=2.6$ in the symmetrical $y 0$-plane. Black lines: $R_{i i}=0.6$. Yellow lines: $R_{i i}=0.0$. Double dashed grey arrows: streamwise length scale (left) and vertical length scale (right).

Based on these correlation coefficient isopleths, the streamwise and vertical size of coherent structures can be estimated. It represents the length of the correlation coefficient profile at a 

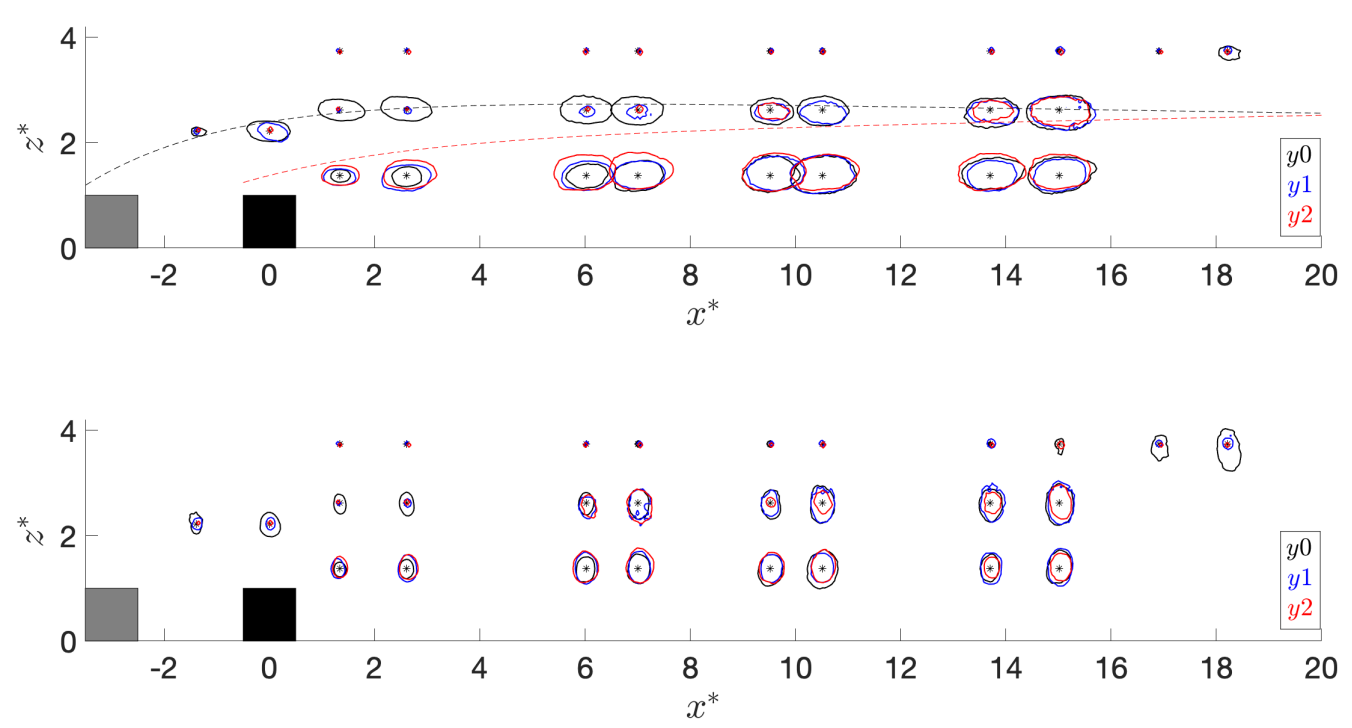

Figure 9: Isopleths of $R_{u u}$ (top) and $R_{w w}$ (bottom) equal to 0.6. Dashed lines ( $y 0$-plane in black and $y 2$-plane in red) on the $R_{u и}$ figure correspond to lines where $\bar{U}=0.90 * U_{\infty}$.

specific point $\left(x_{r e f}^{*}, z_{r e f}^{*}\right)$. This length corresponds to the double arrow indicated in figure 8 , given a qualitative analysis of the flow structures. Furthermore, when the correlation coefficient is inferior to 0.3 , this value is expected to be contaminated by noise and may not be entirely correct. Consequently, it is better to use the present threshold level of 0.6 according to previous work [41]. As the inclination of the structures is small, the length estimation is done along $x$ and $z$ axis. We denote $l_{u_{x}}^{*}$ and $l_{w_{z}}^{*}$ the size of the isopleth ellipses $R_{u u}$ along $x$ and $R_{w w}$ along $z$ respectively. Figure 10 displays the resulting length scales as a function of $z^{*}$ at the $x$-center of the 3 vertical measurement planes. For instance, the integral lengths represented at $x^{*}=2.2$ are deduced from $A, F$ and $J$ PIV measurement planes.

In this figure, upstream one observes that $l_{u_{x}}^{*}=l_{w_{z}}^{*} \approx 0$ above $z^{*}=3.5$ in $y 0$-plane and above $z^{*}=2.5$ in $y 2$ meaning that no significant coherent structures are located in this upper part of the wake flow. At $x^{*}=2.2$ in $y 0$-plane, the highest length scale is obtained at $z^{*}=2.8$ and corresponds to the separated shear layer arising from the cube. A second peak which is a bit weaker, around $z^{*}<1$, is also present. This is due to the cylinder separated shear layer. Downstream $\left(x^{*}=5.7, x^{*}=10\right)$ both peaks evolved similarly and mixed together to form a peak 
plateau for $z^{*} \in[1.6: 2.5]$ at $x^{*}=14$. It is then indicated that the streamwise length scales of energetic structures are significantly impacted by the generation and interaction of turbulent flow structures induced by the presence of the cube. In $y 1$ and $y 2$-planes, even if slight discontinuities appear due to in-between plane measurements, the streamwise length scale follows a similar tendency. In $x^{*}=2.2$, they exhibit one main peak at $z^{*} \approx 1$ (y1-plane) and $z^{*} \approx 1.2$ (y2-plane). Then, their magnitude increases along the streamwise direction and the peak is slightly moved vertically. It then remains constant due to the flow interactions. In $y 0$ and $y 1$-planes, vertical length scales are slowly growing with $x^{*} . l_{w_{z}}^{*}$ remains constant in $y 2$. Whatever the $y^{*}$ value, structures elongate along the streamwise direction.

Globally, in the far wake field, the energetic structures have similar characteristics in each transverse plane strengthening that further downstream, the flow is re-organized and developed mainly in the horizontal direction.

\subsection{Instantaneous vortex identification using the swirling length criterion}

Further to previous works on vortex characterization from 2D database, one proposes to exploit the velocity gradient tensor to access the swirling strength criterion denoted $\Lambda_{c i}[42$, 43]. The imaginary part of the complex eigenvalue of the local velocity gradient tensor $\lambda_{c i}$ is determined [42]. Then, one multiplies $\lambda_{c i}$ to the normalized instantaneous spanwise vorticity to access the direction of rotation of the detected flow structures:

$$
\Lambda_{c i}(x, z)=\lambda_{c i}(x, z) \frac{\omega(x, z)}{|\omega(x, z)|}
$$

Figure 11 displays some representative examples of vortex identification via swirling strength using four consecutive instantaneous 2D PIV snapshots. The top graphs correspond to swirling strength criteria obtained in the measurement plane $A$ just behind the cylinder $x^{*} \in[0: 4.1], z^{*} \in$ [0.7 : 2.2], for both $y 0$ and $y 2$ transverse planes. Both bottom graphs correspond to those computed in the far wake field, in plane $H\left(x^{*} \in[7.5: 12], z^{*} \in[1.5: 3.4]\right)$. Note that the measurement planes do not have the exact same space location.

First, in the near wake field, in $y 2$-plane, vortical structures associated with the shear layer rise slightly just behind the cylinder. These flow structures correspond to energetic Kelvin-Helmholtz instability like those observed in the wake of tabs with a similar vortex identification method [44]. Far away, the shape and orientation of the maximum of swirling strengths are modified. That potentially underlines the appearance of hairpin-like structures [14]. The present planar nature of 

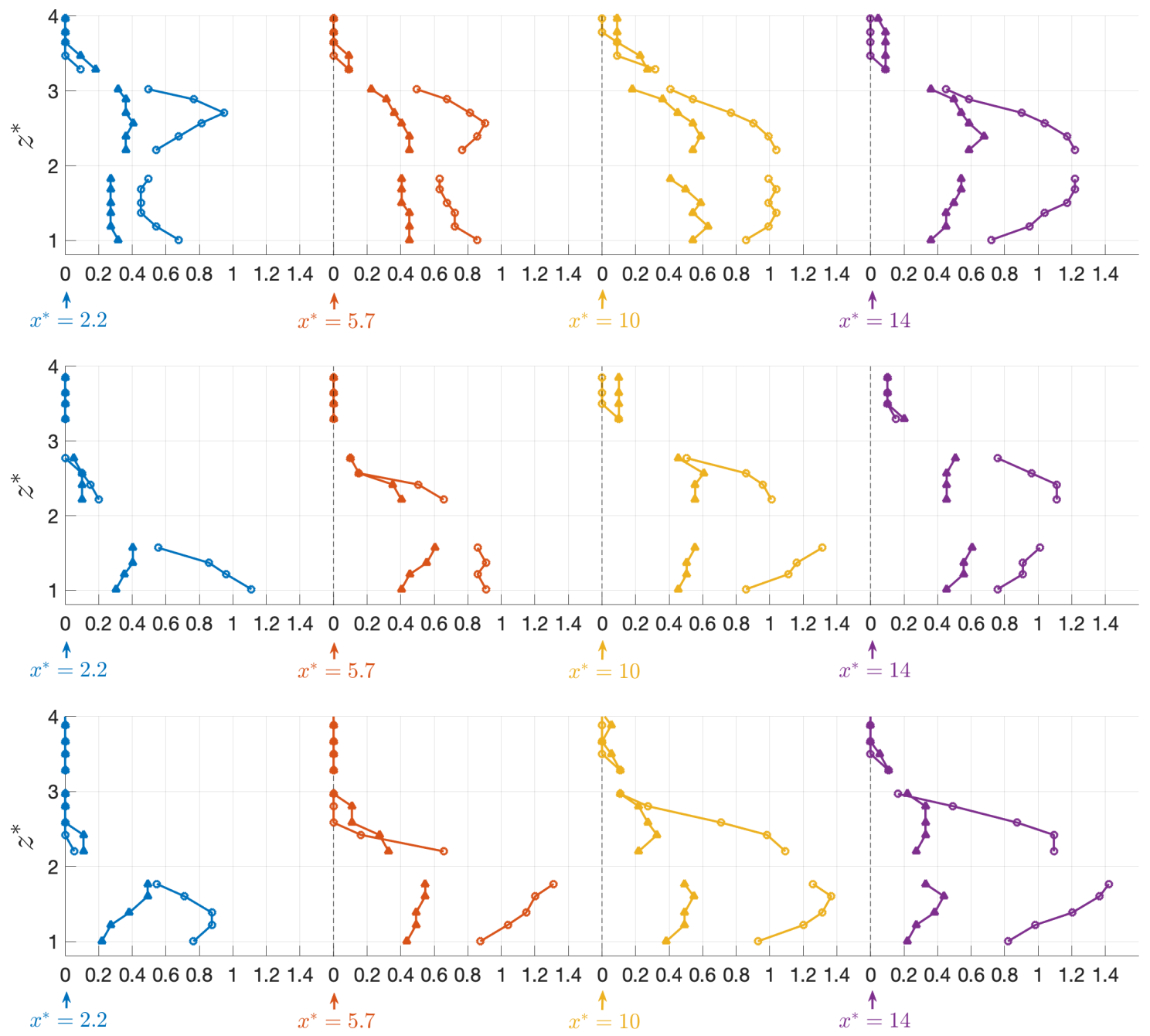

Figure 10: $z$-profiles of the streamwise $\left(l_{u_{x}}^{*} \Longleftrightarrow 0\right)$ and vertical $\left(l_{w_{z}}^{*} \Longleftrightarrow \boldsymbol{\Delta}\right)$ length scales, in four different streamwise positions: $x^{*}=2.2, x^{*}=5.7, x^{*}=10, x^{*}=14$. Top : y0-plane. Center : y1-plane. Bottom : y2-plane.

PIV measurements only enables selected vortical structures to be investigated but does not enable a complex 3D structure like hairpin vortical structures to be investigated.

In the near wake, in the symmetrical $y 0$-plane, the Kelvin-Helmoltz vortices are not easily distinguishable due to the cube wake interaction. A more disturbed flow is observed confirming the $3 \mathrm{D}$ complex nature of the near wake flow in this transverse plane.

In the far wake (bottom graphs), more organized vortical structures with a low frequency content are instantaneously detected in both transverse planes, even if in the symmetrical $y 0$-plane, 
they are more intense. In the $y 2$-plane, the vortical structures could be related to hairpin-like vortices that are advected horizontally in the flow and only located above $z^{*}<2.5$. In the symmetrical $y 0$-plane, vortical structures are also convected parallel to the bottom wall but they are present in a more extented vertical area. This instantaneous analysis confirms previous FFT and statistical results.
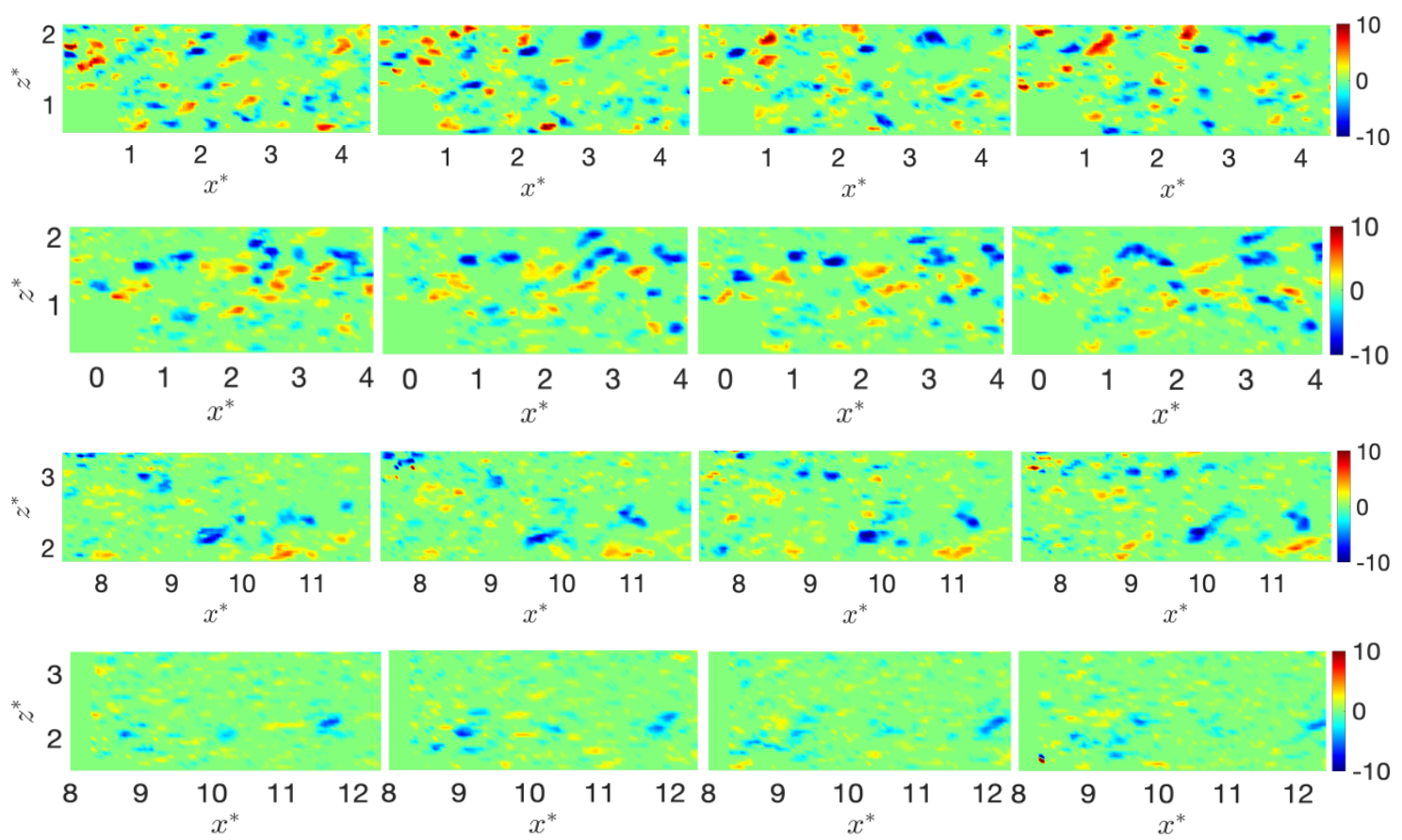

Figure 11: Swirling vortex criterion applied to four consecutive PIV snapshots (from left to right). From top to bottom: Plane A, y0-plane, Plane A, y2-plane, Plane H, y0-plane, Plane H, y2-plane.

\section{Conclusion}

The development of tidal energy devices on a specific area needs the bathymetry induced turbulence to be analyzed first. In this aim, a combination of two obstacles (a cube and a square cylinder) in tandem reproducing at scale a possible seabed roughness has been studied in this work. 2D planar PIV measurements are then carried out in multiple planes to characterize the flow organization in the wake of such a specific tandem configuration. First, it was demonstrated that the near wake is of three dimensional nature and high flow interactions appear especially 
in the symmetrical $y$-plane, leading to some significant modifications of the mean flow characteristics compared to previous published studies dealing with one isolated cube or cylinder configuration. Secondly, a TKE budget analysis has underlined that the TKE is mainly produced in the upstream cube shear layer, and TKE exchanges are small in the far wake field, in comparison with a single cylinder flow configuration. Thirdly, Fourier analyses as well as statistical and instantaneous vortex identification analyses are successively used to investigate the flow organization. It was shown that in the far field, the flow seems to recover a two-dimensional state and large scale energetic flow structures at low frequency are identified. These structures appear as hairpin-like flow structures moving horizontally in the far wake. Indeed, the development of these large scale energetic flow structures in the far field is only limited in a small vertical extent, contrary to the single cylinder wake flow where flow structures rise in the water column.

This works gives further insights into the bathymetry generated turbulence in presence of two different obstacles in tandem. The effect of an upstream cube on the cylinder wake development is clearly highlighted by modifying the near wake and preventing the large scale structure rising in the far wake in the entire water column. The cube induced a notable impact on the wake cylinder structure downstream, especially in the near wake region due to flow interactions. These flow interactions have been analyzed for a specific streamwise spacing between the cube and the cylinder. The dependence of the flow structure on obstacle spacing may be further examined to check at which level of spacing the large scale flow structures are prevented from rising in presence of an upstream cube. Furthermore, as a future work, a tidal turbine could be positioned in the present tandem configuration wake flow and its performance could be compared to those previously obtained in an isolated cylinder wake flow [16, 29]. It will then be possible to identify the most impacting cases on converter behavior, the main concern being if it is an area where an isolated obstacle must be avoided and at what minimal distance to the bathymetry variation the turbine must be deployed. The obtained experimental databases could also be used to validate any other numerical work carried out.

\section{Acknowledgements}

This work benefits from a French state grant managed by the National Research Agency, France under the Investments for the Future program bearing the reference ANR-10-IEED0006-11 (ANR-FEM THYMOTE). This project was partly financially supported by the Euro- 
pean Union (FEDER), the French government, IFREMER and the region Hauts-de-France in the framework of the project CPER 2015-2020 MARCO. The authors would like to gratefully acknowledge Maria Ikhennicheu and Benoit Gaurier involved in the experimental database generation and to thank warmly Thomas Bacchetti and Jean-Valery Facq for their assistance and precious advices.

\section{References}

[1] E. Fernandez-Rodriguez, T. Stallard, S. P.K., Experimental study of extreme thrust on a tidal stream rotor due to turbulent flow and with opposing waves, J. Fluid Struct. 51 (2014) 354-361.

[2] P. Mycek, B. Gaurier, G. Germain, G. Pinon, E. Rivoalen, Experimental study of the turbulence intensity effects on marine current turbines behaviour. part i: one single turbine, Renewable Energy 66 (2014) 729-746.

[3] R. Martinez, G. Payne, T. Bruce, The effects of oblique waves and currents on the loadings and performance of tidal turbines, Ocean Eng. 164 (2018) 55-64.

[4] R. Martinuzzi, C. Tropea, The Flow Around Surface-Mounted, Prismatic Obstacles Placed in a Fully Developed Channel Flow (Data Bank Contribution), J. Fluids Eng. 115 (1) (1993) 85-92.

[5] H. Hussein, R. Martinuzzi, Energy balance for turbulent flow around a surface mounted cube placed in a channel, Phys. Fluids 8 (1996) 764-780.

[6] R. J. Hearst, G. Gomit, B. Ganapathisubramani, Effect of turbulence on the wake of a wall-mounted cube, J. Fluid Mech. 804 (2016) 513-530.

[7] M. Ikhennicheu, B. Gaurier, P. Druault, G. Germain, Experimental analysis of the floor inclination effect on the turbulent wake developing behind a wall mounted cube, Eur. J. Mech. - B/Fluids 72 (2018) 340-352.

[8] C. Diaz-Daniel, S. Laizet, J. Vassilicos, Direct numerical simulations of a wall-attached cube immersed in laminar and turbulent boundary layers, Int. J. Heat Fluid Flow 68 (2017) 269-280.

[9] I. Castro, Relaxing wakes behind surface-mounted obstacles in rough wall boundary layers, J. Fluid Mech. 93 (1979) 631-659.

[10] A. Muller, A. Gyr, On the vortex formation in the mixing layer behind dunes, J. Hydraul. Res. 24 (1987) 359-375.

[11] Y. Liu, F. Ke, H. Sung, Unsteady separated and reattaching turbulent flow over a two-dimensional square rib, J. Fluids Struct. 24 (2008) 366-381.

[12] M. Ikhennicheu, G. Germain, P. Druault, B. Gaurier, Experimental study of coherent flow structures past a wallmounted square cylinder, Ocean Eng. 182 (2019) 137-146.

[13] J. Best, The fluid dynamics of river dunes: a review and some future research directions, J. Geophys. Res. 110 (F4).

[14] P. Mercier, M. Ikhennicheu, S. Guillou, G. Germain, E. Poizot, M. Grondeau, J. Thiébot, P. Druault, The merging of Kelvin-Helmholtz vortices into large coherent flow structures in a high Reynolds number flow past a wall-mounted square cylinder, Ocean Eng. 204 (2020) 107274.

[15] M. Omidyeganeh, U. Piomelli, Large-eddy simulation of two-dimensional dunes in a steady, unidirectional flow., J. Turb. 42 (2011) 1-31. 
[16] B. Gaurier, M. Ikhennicheu, G. Germain, P. Druault, Experimental study of bathymetry generated turbulence on tidal turbine behaviour, Renewable Energy 156 (2020) 1158-1170.

[17] Y. Zhou, M. M. Alam, Wake of two interacting circular cylinders: a review, Int. J. Heat Mass Transfer 62 (2016) 510-537.

[18] D. Sumner, H. Reitenbach, Wake interference effects for two finite cylinders: A brief review and some new measurements, J. Fluids Struct. 89 (2020) 25-38.

[19] M. Zdravkovich, The effects of interference between circular cylinders in cross flow, J. Fluid Struct. 1 (2) (1987) 239-261.

[20] A. Hamed, A. Peterlein, L. Randle, Turbulent boundary layer perturbation by two wall-mounted cylindrical roughness elements arranged in tandem: Effects of spacing and height ratio, Phys. Fluids 31 (2019) 065110.

[21] L. Wang, M. Alam, Y. Zhou, Two tandem cylinders of different diameters in cross-flow: effect of an upstream cylinder on wake dynamics, J. Fluid Mech. 836 (2018) 5-42.

[22] R. Martinuzzi, J. Havel, Turbulent flow around two interfering surface-mounted cubic obstacles in tandem arrangement, J. Fluids Eng. 122 (2000) 24-31.

[23] R. Martinuzzi, J. Havel, Vortex shedding from two surface-mounted cubes in tandem, Int. J. Heat Fluid Flow 25 (3) (2004) 364-372.

[24] G. Yin, M. Andersen, M. Ong, Numerical simulations of flow around two tandem wall-mounted structures at high Reynolds numbers, Applied Ocean Res. 99 (2020) 102124.

[25] B. Gaurier, G. Germain, J.-V. Facq, T. Bacchetti, Wave and current flume tank of IFREMER at Boulogne-sur-mer. Description of the facility and its equipment, Tech. rep., Ifremer (2018). doi:10.13155/58163.

[26] J. Westerweel, F. Scarano, Universal outlier detection for PIV data, Experiments in Fluids 39 (2005) 1096-1100.

[27] T. Kim, K. Christensen, Flow interactions between streamwise-aligned tandem cylinders in turbulent channel flow, AIAA J. 56 (4) (2018) 1-13.

[28] P. Druault, C. Chaillou, Use of Proper Orthogonal Decomposition for reconstructing the 3D in-cylinder mean-flow field from PIV data, C.R. Mecanique 335 (1) (2007) 42-47.

[29] B. Gaurier, P. Druault, M. Ikhennicheu, G. Germain, Experimental analysis of the shear flow effect on tidal turbine performance from three-dimensional mean flow reconstruction, Philosophical Trans. Royal Society A 378 (2020) 20200001.

[30] J. Hinze, Turbulence, 2nd Edition, McGraw-Hill, New York, 1975.

[31] A. Gabriele, A. Nienow, M. Simmons, Use of angle resolved piv to estimate local specific energy dissipation rates for up- and down-pumping pitched blade agitators in a stirred tank, Chemical Eng. Sc. 64 (1) (2009) 126-143.

[32] M. Ikhennicheu, P. Druault, B. Gaurier, G. Germain, Turbulent kinetic energy budget in a wall-mounted cylinder wake using PIV measurements, Ocean Eng. 210 (2020) 107582.

[33] P. Panigrahi, A. Schroeder, J. Kompenhans, Turbulent structures and budgets behind permeable ribs, Exp. Thermal Fluid Sc. 32 (4) (2008) 1011-1033.

[34] A. Kolmogorov, The local structure of turbulence in incompressible viscous fluid for very large Reynolds number, Doklady Akademii Nauk 30 (1941) 9-13.

[35] J. Sheng, H. Meng, R. Fox, A large eddy PIV method for turbulence dissipation rate estimation, Chemical Eng. Sc. 55 (20) (2000) 4423-4434. 
[36] M. Ikhennicheu, G. Germain, P. Druault, B. Gaurier, Experimental investigation of the turbulent wake past real seabed elements for velocity variations characterization in the water column., Int. J. Heat Fluid Flow 78 (2019) 108426.

[37] M. P. Arnal, D. J. Goering, J. A. C. Humphrey, Vortex shedding from a bluff body adjacent to a plane sliding wall, J. Fluids Eng. 113 (3) (1991) 384-398.

[38] P. Krogstad, R. A. Antonia, Structure of turbulent boundary layers on smooth and rough walls, J. Fluid Mech. 277 (1994) $1-21$.

[39] K. T. Christensen, R. J. Adrian, Statistical evidence of hairpin vortex packets in wall turbulence, J. Fluid Mech. 431 (2001) 433-443.

[40] X. Fang, M. Tachie, Spatio-temporal dynamics of flow separation induced by a forward-facing step submerged in a thick turbulent boundary layer, J. Fluid Mech. 892 (2020) A40.

[41] B. Ganapathisubramani, N. Hutchins, W. T. Hambleton, E. K. Longmire, I. Marusic, Investigation of large-scale coherence in a turbulent boundary layer using two-point correlations, J. Fluid Mech. 524 (2005) 57-80.

[42] J. Zhou, R. J. Adrian, S. Balachandar, T. M. Kendall, Mechanisms for generating coherent packets of hairpin vortices in channel flow, J. Fluid Mech. 387 (1999) 353-396.

[43] Y. Wu, K. Christensen, Population trends of spanwise vortices in wall turbulence, J. Fluid Mech. 568 (2006) 55-76.

[44] A. Hamed, A. Pagan-Vazquez, D. Khovalyg, Z. Zhang, L. P. Chamorro, Vortical structures in the near wake of tabs with various geometries, J. Fluid Mech. 825 (2017) 167-188. 